\author{
Daniel Giltard \\ Conseiller d'État honoraire
}

\title{
L'IDÉE D'ÉTAT
}

UDK: 340.12

Primljeno: 1. rujna 2018.

Izvorni znanstveni rad

Quand on parle de l'Etat, c'est plus pour dire ce qu'il fait, les politiques publiques qu'il met en oeuvre, que ce qu'il est. L'Etat nous est ou nous semble familier. Nous n'éprouvons pas le besoin de nous poser les questions : qu'est-ce que l'Etat, d'où vient-il ? Nous avons au moins deux bonnes raisons pour ne pas nous poser ces questions.

L'une est " l'amnésie de la genèse » de toute institution réussie, nous dit le sociologue Pierre Bourdieu. Une institution est réussie lorsqu'elle a réussi à s'imposer comme allant de soi, à faire oublier sa genèse. L'institution réussie fait oublier qu'elle a eu une naissance, un commencement. Elle existe. C'est ainsi, c'est comme çà. On ne se pose pas de questions sur ses origines. C'est le cas pour l'Etat, qui a été institutionnalisé. Une autre raison est que l'Etat, ce qu'il est, n'est pas enseigné. Ce sont les questions de gestion, de management qui ont monopolisé l'attention, mais la réflexion évolue. Il y a une abondante littérature sur l'Etat, émanant de juristes, sociologues, philosophes, économistes, historiens. Pierre Rosanvallon, en annexe à son livre « L'Etat en France entre 1789 et nos jours ", publié en 1990, présentait sur le thème de l'Etat une bibliographie de près de 2000 titres et depuis cette date de nombreux ouvrages ont été publiés. Récemment le directeur de l'Ecole nationale d'administration écrivait ceci : " L'ENA doit d'abord être une école où, au-delà du management, sont enseignés l'Etat, le rôle de l'Etat, le sens de l'Etat » Les réformes de l'Etat, de ses missions, de son organisation, doivent être préparées par une réflexion sur ce qu'est l'Etat. C'est dans ce but qu'a été organisé par le Conseil d'Etat de 2013 à 2015 un cycle de conférences sur le thème " Où va l'Etat? ", avec une première conférence sur le concept d'Etat, l'Etat comme objet de philosophie politique et comme construction historique. C'est la question : qu'est-ce que l'Etat ? Dans son discours introductif à ce cycle de conférences, le Vice-président du Conseil d'Etat, JeanMarc Sauvé, rapporte une anecdote significative sur l'importance de la philosophie de l'Etat. Une délégation des élèves de l'Ecole nationale d'administration rend visite en 1976 à notre célèbre écrivain et ancien ministre André Malraux. Les élèves lui présentent les divers enseignements suivis à l'Ecole. C'est alors qu'André Malraux s'esclaffe « Mais enfin vous préparez un diplôme de technicien. Ce qu'il faut enseigner à l'ENA, ce sont des cours sur la problématique et la philosophie de l'Etat » $\mathrm{Il}$ y a des définitions bien connues de l'Etat.

Une première définition nous a été donnée par les juristes allemands et français à la fin du $19^{\text {ème }}$ siècle « L'Etat est la nation juridiquement organisée » Dans une version plus moderne, on parle de l'Etat comme de « la société juridiquement organisée » En effet la Nation n'a pas toujours précédé l'Etat. Par exemple en France, c'est l'Etat qui a produit, construit la Nation. Il y a aussi cette autre définition classique des juristes. L'Etat, c'est un territoire, une population, une autorité souveraine et on ajoute parfois un quatrième critère, la reconnaissance en tant qu'Etat par la communauté internationale. Mais l'Etat, est-ce cela ? ou plutôt n'est-ce que cela ?

Mots clés: L'etat, concept, France 


\section{L'ÉTAT EST UNE INVENTION DE L'ESPRIT}

Puisqu'il s'agit d'une définition juridique, interrogeons d'abord un juriste

Dans son livre intitulé « L'Etat», publié en 1970, Georges Burdeau commence par nous dire : "Personne n'a jamais vu l'Etat. Qui pourrait nier cependant qu'il soit une réalité » L'auteur convient qu'il n'y a certes pas d'Etat sans territoire, sans population et sans autorité qui commande, mais, ajoute-t-il, il faut distinguer, dans une approche philosophique, l'existence de l'Etat de son essence.

Les phénomènes concrets, ce que l'on voit, le territoire, la population, les services, les gouvernants ne font que révéler l'existence de l'Etat. Ils ne renseignent pas sur son essence.

L'Etat est une construction de l'esprit, une idée qui transcende ces données sensibles. Il est une réalité conceptuelle.

Qu'en pensent les sociologues?

Pierre Bourdieu partage cette conception abstraite de l'Etat. L'Etat, du moins l'Etat occidental, est une fiction, une construction, une invention de l'esprit, un concept. " L' Etat, on l'a souvent dit, est une fictio juris. C'est vrai, mais c'est une fiction de juristes, en donnant à fictio le sens fort du terme, de fingere ( construire », " fabriquer ») : c'est une fabrication, une construction, une conception, une invention»

Un autre auteur, Patrick Le Galès, reprend la même idée L'Etat est " un récit, une abstraction, une fiction », mais aussi, et l'expression caractérise bien l'Etat « un grand mythe unificateur »

\section{LA GENÈSE DU CONCEPT D'ETAT}

Mais alors on se pose des questions sur la genèse de ce concept : quand, où, qui, comment, pourquoi ?

\section{Quand le concept d'Etat a-t-il été créé ?}

A l'époque médiévale, du $11^{\text {ème }}$ au $13^{\text {ème }}$ siècle.

\section{Où ?}

En Europe, à commencer par l'Angleterre, puis la France, la France de cette époque, un royaume féodal que l'on commence à appeler France au $12^{\text {ème }}$ siècle.

Nous parlons donc de l'Etat de notre Europe occidentale. Il faut toutefois noter que l'Angleterre s'est écartée du concept abstrait de l'Etat et a développé au $16^{\text {ème }}$ 
siècle une fiction juridique particulière, quasi-théologique, la fiction des « Deux Corps du Roi », qui s'inspire de la notion romaine de « corporatio»

\section{Par qui le concept d'Etat a-t-il été créé?}

Par les juristes.

L'Etat est une fiction juridique

\section{Pourquoi ce sont les juristes qui ont fabriqué cette fiction, ce concept d'Etat?}

Avec le vocabulaire d'aujourd'hui, certes anachronique, mais parlant, on dirait que dans la société de cette époque, il y avait de grandes catégories socioprofessionnelles, socio-culturelles, qui, chacune dans son champ d'activités, qu'il s'agisse du champ économique, culturel, militaire, administratif, juridique, avait un capital particulier de connaissances, de compétences, de savoirs, d'informations et avait aussi un point de vue sur l'organisation de la société. Ces différents acteurs de la société étaient non seulement en concurrence entre eux, mais surtout en lutte pour l'exercice de la domination sur l'ensemble de la société.

Dans cette lutte pour la domination de la société, les juristes l'ont emporté parce qu'ils avaient le capital le mieux adapté, le capital juridique, hérité du droit romain, un capital de mots et de concepts, auxquels ils savent donner une valeur universelle, une force officielle, une valeur symbolique. Par ce capital de mots et de concepts, ils construisent la réalité. Les juristes défendaient leurs intérêts particuliers, leur place dans la société, auprès du Prince, mais ils avaient l'outil conceptuel pour imaginer cette abstraction qu'est l'Etat, cette fiction qui facilite l'exercice du pouvoir.

Il est en effet plus facile pour les hommes d'obéir à une institution impersonnelle, désincarnée, plutôt que d'être soumis à la domination directe d'autres hommes.

Grace à cette fiction, c'est en effet l'Etat qui décide, demande, promet, s'engage, agit. Les officiels parlent au nom de l'Etat. C'est la figure rhétorique de la prosopopée, mais je préfère l'expression de Bourdieu «l'homme officiel est un ventriloque qui parle au nom de l'Etat »

L'Etat est personnifié, on lui prête un visage. Les figures données à l'Etat sont multiples. Les principales sont les figures de l'Etat gendarme, de l'Etat providence, de l'Etat stratège, régulateur, aujourd'hui.

\section{Comment le concept d'Etat a-t-il été construit ?}

L'Etat est une rupture, mais, en même temps, un héritage et une construction continue. 
Avant de parler de ces trois aspects de la construction du concept d'Etat, disons, de façon générale, que les deux mots qui caractérisent l'Etat, dans ses relations avec la société, sont la souveraineté et la solidarité. Ce fut d'abord par la souveraineté, qui est consubstantielle au concept même d'Etat, puis aussi, mais plus tard, par la solidarité, que l'Etat fait tenir debout la société, fait vivre ensemble une multitude d'individus.

\section{L'ETAT EST UNE RUPTURE}

Rupture par rapport à un système, le système féodal de dépendance personnelle, d'allégeance des vassaux à leur suzerain. On passe de la suzeraineté féodale à la souveraineté royale, qui s'exprime par l'édiction de règles. L'Etat libère les hommes de tout lien de dépendance personnelle. Ils sont soumis aux mêmes lois générales et abstraites. Apparaissent des notions caractéristiques de l'Etat. La souveraineté royale s'exerce sur un territoire et est fondée par les légistes sur l'idée que le prince agit en vue du «commun profit » et de «l'utilité publique »

Si je devais proposer la date de naissance de l'Etat en France, je dirais : l'an 1190. Le roi Philippe Auguste, qui va partir en croisade, prend une ordonnance fixant l'organisation du royaume pendant son absence. On appelle cette ordonnance le « testament » de Philippe Auguste, car on n'était jamais sûr de revenir d'une croisade ! Ce texte met en place une administration centralisée et hiérarchisée sur l'ensemble du territoire du royaume. Le roi devient souverain d'un territoire et cette souveraineté est justifiée par l'utilité publique. L'ordonnance commence par cette proclamation : " : L'office du roi consiste à pourvoir par tous les moyens aux besoins de ses sujets, et à faire passer l'utilité publique avant sa propre utilité »

Quelques années plus tard, en 1204, le roi, qui était le roi des Francs, de l'un des peuples qui vivaient dans le royaume, s'intitule roi de France, montrant ainsi que le pouvoir monarchique a désormais une base territoriale.

\section{L'ETAT EST UN HÉRITAGE}

Il y a en réalité plusieurs héritages.

Un héritage philosophique. Cette volonté de soumettre toutes les personnes aux mêmes lois est l'héritage de l'idéal grec de la cité régie par la loi. Depuis Aristote, nous souhaitons être gouvernés « par les lois et non par les hommes » Or c'est à cette époque médiévale des $12^{\text {ème }}$ et $13^{\text {ème }}$ siècles que l'Université naissante a redécouvert la pensée d'Aristote grâce la traduction de ses textes rédigés en langue arabe et qui avaient été conservés par les émirs andalous.

Un héritage juridique. 
Le concept même d'Etat n'a pas été construit ex nihilo par les juristes de l'époque médiévale. Ils se sont inspirés de l'Eglise, de son organisation, du droit canonique, mais aussi du droit romain.

Les canonistes sont d'ailleurs souvent présentés comme les inventeurs de l'Etat moderne et on parle parfois de l'Etat comme d'une « religion civile » (Pierre Bourdieu)

Mais pourquoi les juristes ont-ils pris tant d'importance aux $11^{\text {ème }}$ et $12^{\text {ème }}$ siècles?

Pour deux raisons. Deux évènements majeurs ont eu lieu à cette époque, l'un a créé un besoin de droit, l'autre a proposé une nouvelle offre de droit. Les juristes ont alors eu un rôle croissant. Le $12^{\text {ème }}$ siècle est le siècle de la renaissance juridique (par exemple, distinction par les théologiens, reprise par les juristes, entre le droit naturel et le droit positif ; distinction entre actes publics et actes privés, à l'origine d'une théorie de l'acte authentique, très liée à l'autorité de l'Etat )

Le premier évènement est considérable. C'est ce qu'on appelle la réforme « grégorienne », du nom du pape Grégoire VII (1073-1085), véritable révolution culturelle qui a profondément modifié nos sociétés occidentales, par la nette séparation entre les clercs et les laïcs et la primauté du pouvoir pontifical au sein de l'Eglise.

La chute de l'Empire romain d'Occident en 476 avait ouvert un vide institutionnel, qui a été comblé au Moyen-Age par l'Eglise catholique. S'est posée alors la question du pouvoir de la papauté. En 1075 le pape Grégoire VII, dans son dictatus papae, formule un ensemble de propositions sur les pouvoirs du pape. Parmi ces propositions, il y a la distinction entre l'autorité spirituelle et le pouvoir temporel et l'affirmation du pouvoir souverain du pape sur l'administration de l'Eglise. Cette reconnaissance d'un pouvoir temporel souverain a servi de base à la création de notre Etat moderne.

Un fort besoin de droit s'exprime, tant de la part de l'Eglise, devenue une institution avec un gouvernement central, que de ceux qui contestent ses nouveaux pouvoirs. Le droit canonique se développe au-delà des questions religieuses, traitant du droit des personnes. Des compilations des textes canoniques, ce que l'on appelle des « sommes » sont établies pour l'enseignement (la « somme» la plus connue est le Décret de Maître Gratien, moine italien, commencé vers 1140 à Bologne, puis remanié et complété vers 1160).

Un autre évènement important à cette même époque propose une nouvelle offre de droit.

C'est la redécouverte entre Rome, Ravenne (Ravenne et son célèbre «banquet»), Sienne et Pise des manuscrits des célèbres compilations de l'Empereur Justinien. A côté du droit canonique apparaît un droit nouveau avec cette redécouverte du droit romain. Les juristes vont se servir de ce nouveau droit.

Sont notamment reprises du droit romain la notion de jus publicum et la distinction entre le droit public et le droit privé, qui est issue des travaux d'Ulpien, 
juriste romain du IIIème siècle, repris dans le Digeste. Ulpien distinguait le droit public qui ne concernait que l'état, le status, de la chose romaine, c'est -à- dire de la res publica, et le droit privé qui concernait les rapports entre les particuliers. Le droit public était le droit vertical. Il fallait que la chose publique, la res publica tienne debout, soit en position verticale, pour que les rapports horizontaux entre les particuliers obéissent à un régime de droit et non à la loi du plus fort. L'Etat moderne est aussi l'héritier de « l'état de la chose romaine»

On vient de parler d'état ou de status. On sait que le mot état a pour étymologie le mot latin status, qui vient du verbe stare, se tenir debout. Ce mot de status ou d'état a été appliqué à tous les grands pouvoirs institués pour tenir et faire tenir debout une société humaine, aussi bien à l'Empire romain, qu'à l'Eglise ou à la Monarchie, mais le mot était toujours complété par l'institution à laquelle il s'appliquait. C'étaient le status Imperii, le status Ecclesiae, le status Regni. L'état de l'Empire, de l'Eglise, de la Monarchie, c'est-à-dire la manière dont ces institutions existaient et subsistaient. L'état ainsi entendu était propre à chaque institution.

Les juristes du Moyen-Age ont supprimé tout complément pour faire de l'état une fiction juridique, un concept qui se suffit à lui-même, qui a une valeur universelle, le concept d'Etat. Non plus l'état des personnes, des institutions, mais l'Etat tout court, que l'on a écrit avec un E majuscule, la majuscule de la majesté, pour signifier sa valeur symbolique, son caractère officiel. C'est l'Etat majuscule, par rapport à l'état minuscule, l'état des personnes, l'état civil.

\section{Quelles sont les principales caractéristiques de l'Etat créé par les juristes?}

C'est un Etat souverain, légal, vertical, centralisé, dominateur.

Plus précisément, c'est un concept fabriqué par les juristes pour permettre au roi d'exercer un pouvoir souverain (l'Etat souverain) sur l'ensemble du territoire de son royaume, au moyen de règles générales et impersonnelles édictées au nom de l'Etat ( l'Etat légal) et appliquées par un appareil d'Etat organisé de façon verticale et centralisée ( L'Etat vertical et centralisé)

L'Etat est centralisé, car sa construction s'est faite par un processus de concentration, de monopolisation au niveau central, dans une organisation qui est devenue rationnelle et bureaucratique, des différentes espèces de capital de connaissances, de compétences.

A ce processus de concentration, de monopolisation des connaissances et des savoirs au niveau central s'est ajoutée une centralisation administrative. L'Etat créait ainsi sur l'ensemble du territoire, de façon visible, des liens de type vertical, sans intermédiaire, entre le pouvoir souverain et le peuple. Ce sont des liens de dépendance uniformes, fondés sur des règles.

L'Etat centralisé est donc aussi un Etat légal, mais c'est surtout un Etat construit dans un esprit de domination. L'Etat est devenu le pouvoir sur les pouvoirs, le point de vue des points de vue. C'est le pouvoir qui est au-dessus, en surplomb des autres 
pouvoirs, qui est dans une position d'extériorité et de supériorité par rapport à la société, qui la domine, qui est fondé sur l'idée de puissance inconditionnée

L'Etat a le monopole de la violence physique légitime, selon la formule célèbre de Max Weber. La définition de l'Etat donnée par Max Weber est plus complète, mais c'est «le monopole de la violence physique légitime » qui en est la notion essentielle.

Mais l'Etat est devenu à la fin du $19^{\text {ème }}$ siècle et au début du $20^{\text {ème }}$ un Etat de droit et un Etat social.

De grands débats ont eu lieu à cette époque entre juristes allemands et juristes français, et au sein même des juristes français, sur la question de la personnalité morale reconnue à l'Etat, qui donne à l'Etat le pouvoir subjectif de commander, en fait le siège d'un pouvoir inconditionné et sur la nécessaire limitation de l'Etat.

L'Etat est soumis au droit. C'est un Etat de droit, mais la doctrine publiciste allemande, Rudolph Von Jhering, Paul Laband et Georg Jellinek, défendait la thèse de l'autolimitation de L'Etat. L'Etat est certes un Etat de droit, mais, comme il détient seul la puissance de contrainte, il est la seule source de droit. Le droit n'est donc pas une limite extrinsèque. C'est l'Etat lui-même qui, par sa propre volonté de personne morale, se soumet au droit qu'il crée.

Le concept d'Etat de droit a été repris par les juristes français au début du $20^{\text {ème }}$ siècle, mais la thèse allemande de l'autolimitation a fait l'objet de vives critiques. C'est la question fondamentale des rapports entre l'Etat et le droit. Le droit a-t-il sa source dans l'Etat ou est-il antérieur, extérieur, supérieur à l'Etat ?

La position dominante de la doctrine française était de soumettre l'Etat au droit. Les grands juristes du début du $20^{\text {ème }}$ siècle, notamment Léon Duguit et Maurice Hauriou, ont construit, en s'inspirant de la sociologie, de la philosophie, des théories destinées à limiter la puissance de l'Etat par le droit. L'Etat doit être soumis au droit et non s'y soumettre librement. Le droit est extérieur et supérieur à l'Etat. Il faut, écrit Hauriou "emprisonner l'Etat dans les mailles du filet juridique » Dans la société l'Etat n'est pas au-dessus, il est une institution, certes la plus importante, "l'institution des institutions », dit Hauriou, mais il n'est qu'une institution et la personnalité de l'Etat n'est qu'une personnalité juridique et non une personnalité métaphysique, transcendantale. l'Etat est un sujet de droit. Il est descendu de son piedestal, c'est « l'Etat détrôné » ( Fabrice Melleray). Il est, comme toute personne, comme toute institution, soumis aux règles de droit.

C'"est toutefois l'introduction dans notre droit du concept de service public qui a été l'élément essentiel de la limitation de la puissance de l'Etat. La puissance de l'Etat n'est en effet justifiée qu'autant qu'elle est nécessaire pour répondre aux besoins collectifs du public, pour exercer les missions de service public qui incombent à l'Etat.

Il ne faut toutefois pas se méprendre sur la portée de la formule de Max Weber. La violence ou la contrainte physique n'est pas pour Weber le moyen normal d'action 
de l'Etat. Ce n'est que l'ultima ratio, le dernier recours pour imposer l'obéissance à la loi.

Il n'est, heureusement, pas toujours besoin de recourir à la contrainte physique pour que les gens obéissent, respectent les règles.

L'Etat s'impose parce que, ce qu'il fait, ce qu'il dit, est marqué du sceau de l'officiel et du symbolique. L'Etat repose sur la confiance, la croyance, sur la représentation que les gens s'en font. Or, dans cette représentation, un certain nombre d'institutions, de pratiques ont acquis une valeur symbolique et sont acceptées comme une évidence. A ce pouvoir symbolique de l'Etat s'ajoute la force de l'officiel. L'Etat a le monopole de l'officiel. A son contact, les personnes, les mots, les choses acquièrent un caractère officiel : les personnages officiels, les textes officiels, les titres officiels, le journal officiel, les discours officiels etc...

Chacun d'entre nous se fait sa propre représentation de l'Etat, mais l'Etat participe à sa représentation par la mise en scène, la théâtralisation que constituent les cérémonies officielles, les rituels, les commémorations symboliques.

"Il faut du théâtre, des rites, des cérémonies d'écriture pour faire exister un Etat, lui donner forme, en faire une fiction animée », écrit Pierre Legendre dans «Miroir d'une Nation»

\section{L'ETAT EST UNE CONSTRUCTION CONTINUE}

Le concept d'Etat a été construit par différents emprunts, au droit romain, au droit canonique, mais ce n'est pas un concept figé. La construction de l'Etat s'est poursuivie au cours des siècles par «accumulation de sédiments » par « collages » successifs et notre Etat moderne est «un produit de synthèse » (Pierre Legendre ) Rien ne se perd et notre Etat d'aujourd'hui, post-moderne dit-on, ne peut gommer ce qu'il doit à tous ces sédiments, ces collages depuis le droit romain, mais l'Etat continue de se faire, s'adapte aux évolutions sociales, économiques et politiques.

L'Etat ne se définit que par rapport à la société. Il a pour fonction, on l'a dit, de faire tenir debout la société, de lui donner cohésion et solidité, de faire tenir ensemble les individus dans une même société, mais la société n'est pas restée passive. Elle a contribué elle-même à sa solidité en organisant des réseaux de solidarité.

Il est significatif de noter que, pour les juristes des $17^{\text {ème }}$ et $18^{\text {ème }}$ siècles, les mots de solidarité et de solidité étaient synonymes.

La solidarité était l'affaire des individus : solidarité familiale, solidarité de voisinage, d'affinités, solidarité au sein des métiers, des corporations, des confréries.

Puis l'Etat a été amené à prendre en charge la solidarité sociale. L'Etat souverain est devenu aussi Etat social.

La raison de cette évolution : la révolution industrielle au cours du $19^{\text {ème }}$ siècle. L'industrialisation et l'urbanisation ont affaibli les solidarités civiles traditionnelles et c'est l'Etat qui progressivement a pris en charge directement la solidarité sociale. 
Cela a commencé avec les lois sur les assurances sociales de l'Allemagne de Bismarck à la fin du $19^{\text {ème }}$ siècle et en France avec la loi du 9 avril 1898 sur les accidents du travail

L'Etat social a été présenté comme "la grande invention institutionnelle du XX $X^{\text {ème }}$ siècle » (Alain Supiot)

L'Etat a en effet été transformé. Il n'est plus seulement la puissance extérieure, qui domine, qui prescrit. Il est devenu un acteur du jeu social. il agit pour le bienêtre des hommes par ses services publics. Les prescriptions ont fait place aux prestations. Il a pris la figure, selon les pays, de l'Etat providence, du Welfare state, du Sozialstaat, de la République sociale.

Cette évolution de l'Etat répondait certes au besoin de pallier le délitement des solidarités civiles traditionnelles, mais elle a été aussi et surtout en France un choix de philosophie politique pour donner un fondement conceptuel, une légitimité à l'Etat républicain de la fin du $19^{\mathrm{ème}}$ siècle et du début du $20^{\text {ème }}$.

L'Etat intervenait de plus en plus dans la vie économique et sociale. Cette intervention était contestée, aussi bien par les libéraux, qui voulaient limiter l'Etat à ses fonctions régaliennes, que par les socialistes qui voulaient limiter les interventions de l'Etat « bourgeois».

C'est le concept de solidarité qui est venu en justification de l'intervention de l'Etat dans le domaine économique et social.

La doctrine de la solidarité ou de l'interdépendance sociale, chère à Léon Duguit, qui emprunte aux travaux des sociologues, notamment d'Emile Durkheim, mais aussi des philosophes, des économistes, sans oublier l'apport des recherches de Louis Pasteur, de " la révolution pasteurienne » ( Pierre Rosanvallon), était très en vogue à la fin du $19^{\text {ème }}$ siècle. Elle a inspiré une nouvelle philosophie politique, le solidarisme (auquel est attaché le nom de Léon Bourgeois ), qui a donné une nouvelle légitimité à l'Etat. Si l'Etat intervenait dans la vie économique et sociale, c'était pour assurer cette solidarité sociale.

Le service public est le prolongement, la traduction juridique de la solidarité. C'est par ses services publics que l'Etat garantit la solidarité sociale.

Pour reprendre la célèbre formule de Léon Duguit " l' Etat n'est pas une puissance qui commande, une souveraineté, il est une coopération de services publics organisés et contrôlés par des gouvernants »

L'Etat social figure dans la plupart des Constitutions des Etats européens. Nos Constitutions, dès l'article $1^{\text {er }}$, prévoient que la France est une République sociale, que la République de Croatie est un Etat social.

Cet Etat social a été contesté, remis en cause à partir des années 1980. Le service public a été critiqué, sa gestion jugée peu efficace, coûteuse. Le New Public Management a entendu soumettre l'administration publique aux modes de gestion de l'entreprise privée, avec des objectifs de performance. On est passé du gouvernement par la loi à «la gouvernance par les nombres» (Alain Supiot) 
Mais d'autres causes ont contribué à l'évolution de l'Etat, qui vit désormais dans une situation d'interdépendance avec d'autres acteurs publics ou privés, internes ou internationaux. C'est l'effet de l'européanisation, de la mondialisation, de la globalisation. En France c'est aussi la décentralisation des années 1980 et de nouveaux modes de gestion, notamment la multiplication des agences. La révolution numérique oblige aujourd'hui à réfléchir à de nouvelles formes de management et a une incidence sur la place de l'Etat.

Le constat est que l'Etat est devenu modeste, mais évidemment ce n'est pas la fin de l'Etat.

L'Etat a montré au contraire sa capacité de résilience et d'adaptation. L'Etat central s'est affaibli, mais l'Etat s'est adapté, il vit autrement, c'est « l'Etat recomposé ». Il est devenu un Etat stratège, régulateur, dans un rôle d'impulsion et de contrôle. C'est l'ère du polycentrisme. L'Etat vit en réseau avec d'autres centres de décision publics ou privés.

Ce n'est plus l'Etat central des juristes, qui depuis le début du $20^{\text {ème }}$ siècle, portait les solidarités sociales, qui s'était substitué aux solidarités civiles traditionnelles. Si l'Etat est conduit à se désengager, quel est l'avenir de la solidarité sociale, donc de la solidité de la société?

L'idée générale est de savoir s'il va y avoir un mouvement naturel de balancier entre, d'une part, les solidarités traditionnelles, familiales, territoriales, professionnelles, d'autre part, l'Etat social.

Depuis la fin du $19^{\text {ème }}$ siècle les solidarités traditionnelles se sont affaiblies du fait de la prise en charge des solidarités sociales par l'Etat qui avait un véritable monopole. Aujourd'hui c'est l'Etat social qui est affaibli. Va-t-on assister à un retour des solidarités traditionnelles ou à la naissance d'autres formes de solidarité, religieuses, communautaires ? Plusieurs chercheurs (Pierre Legendre, Alain Supiot) nous alertent sur le risque de voir se constituer des réseaux d'allégeance personnelle de type féodal, où chacun recherche la protection de plus fort que lui, dans des réseaux d'appartenances claniques, religieuses, ethniques ou même mafieuses.

L'Etat reste fondamental pour la cohésion sociale, il est le garant de la solidarité nationale, mais son rôle est difficile. Il doit prendre en compte la constitution de ces réseaux d'allégeance personnelle et est enserré lui-même dans un vaste réseau, interne et international, de décideurs publics et privés. 


\section{Principales sources bibliographiques}

Blanquer Jean-Michel, Millet Marc : «L'Invention de l'Etat», Editions Odile Jacob 2015

Bourdieu Pierre : «Sur l'Etat », Cours au Collège de France, 1989-1992, Editions du Seuil 2012

Braud Philippe: « Penser l'Etat » Editions du Seuil, 2004

Burdeau Georges : «l'Etat » Editions du Seuil, 2009, avec préface de Philippe Braud

Caillosse Jacques : «L'Etat du droit administratif », LGDJ 2015

Cassard Jean-Christophe : «L'âge d'or capétien, 1180-1328 » Belin

Chevalier Jacques : « Le service public » édition 2010, Presses universitaires de France.

Chevalier Jacques: « L'Etat de droit » Editions Montchrestien, 1994

Conseil d'Etat, Droits et Débats, « Où va l'Etat ? » Cycle de conférences au Conseil d'Etat, La documentation française, tome 1, 2015, tome 2, 2016

Denoix de Saint Marc Renaud: « L'Etat », Presses universitaires de France 2004

Kantorowicz Ernst : «Les Deux Corps du Roi, essai sur la théologie politique au moyen âge » Editions Gallimard Quarto, 2000.

Le Galès Patrick, Vezinat Nadège, « l'Etat recomposé », Presses universitaires de France, 2014

Legendre Pierre : « Miroir d'une Nation, l'Ecole Nationale d'Administration » et « Les collages qui font un Etat », Editions Mille et une nuits, 1999

Mazel Florian : «Féodalités, 888- $1180 »$, Belin

Melleray Fabrice : «Léon Duguit. L'Etat détrôné », in Le renouveau de la doctrine française, Editions Dalloz 2009

Rosanvallon Pierre: « L'Etat en France de 1789 à nos jours », Editions du Seuil 1990

Supiot Alain, « Grandeur et misère de l'Etat social », Leçon inaugurale au Collège de France du 29 novembre 2012, Editions Fayard

Supiot Alain « La gouvernance par les nombres »Cours au Collège de France ( 20122014), Editions Fayard 


\section{THE CONCEPT OF STATE}

When the idea of state is discussed, that discussion exceeds the framework of state activity. That is, in that case, the public policies which a state produces must also be considered. Here the author stresses that there are several reasons why often there is no need to pose the questions of what is state and from where does state originate? The opinion of sociologist Pierre Bourdieu is given as one reason. He emphasizes ,amnesia of genesis "of any successful institution. Namely, according to the opinion of Pierre Bourdieu, an institution is successful when it succeeds in imposing itself as obvious and thereby does not raise the question of its origin. An institutionalized state simply exists. State reform, its missions and organization, must be prepared intentionally according to what makes a state. Bearing this in mind, the State Council from 2013 to 2015 organized a few conferences on the topic: „Where is the State? “, the conclusion to which is provided in this article. Furthermore, the author poses the question on the inception of this very concept of: when, where, who, how, why? How was the concept of state created? What are, according to lawyers' opinion, the main characteristics of a state? In addition, the author attempts to offer answers to the questions raised, emphasizing how the state is a continuous creation.

Key words: state, concept, France

\section{POJAM DRŽAVE}

Svaka rasprava o ideji države nadilazi okvire državne aktivnosti. Ako je to slučaj, onda se mora voditi računa o javnim politikama koje država proizvodi. Autor naglašava da postoji nekoliko razloga zašto često nema potrebe postavljati pitanja o tome što je i odakle potječe država? Mišljenje sociologa Pierrea Bourdieua dano je kao jedan od razloga. Naglašava se „amnezija geneze“ svake uspješne institucije. Naime, prema mišljenju P. Bourdieua, institucija je uspješna kada se uspije nametnuti kao očigledna i time ne postavlja pitanje njezina podrijetla. Institucionalizirano stanje jednostavno postoji. Državna reforma, njezine misije i organizacija moraju se namjerno pripremati prema onome što čini državu. Imajući to u vidu, Državno vijeće je od 2013. do 2015. godine organiziralo nekoliko konferencija na temu: „Gdje je država?“ O tome se govori i u ovomwe tekstu. Nadalje, autor postavlja pitanje o nastanku samog pojma: kada, gdje, tko, kako, zašto? Kako je stvoren koncept države? Što su, prema mišljenju odvjetnika, glavne značajke države? U svjim odgovorima autor naglašava da je država kontinuirana tvorevina.

Ključne riječi: država, koncept, Francuska 